\title{
Pronto atendimento a usuários de dispositivos de amplificação sonora
}

\section{Emergency care to users of hearing aids}

\author{
Tiago de Melo Araujo ${ }^{1}$, Beatriz de Castro Andrade Mendes $^{2}$, Beatriz Cavalcanti de Albuquerque Caiuby Novaes ${ }^{3}$
}

\begin{abstract}
RESUMO
Objetivo: Estabelecer características demográficas e audiológicas de sujeitos usuários de aparelhos de amplificação sonora individuais (AASI) que solicitaram retorno em um Serviço de Saúde Auditiva e identificar e analisar os motivos do retorno e condutas geradas durante o Pronto Atendimento (PA). Métodos: Os participantes foram 440 sujeitos de idade superior a três anos, com diagnóstico e concessão do AASI no serviço onde foi realizada a pesquisa. Características demográficas, audiológicas e referentes ao uso do AASI foram coletadas em prontuários e ficha específica para o PA. Os dados obtidos foram analisados por meio de estatística descritiva e inferencial. Resultados: O grupo estudado foi caracterizado, em sua maioria, por indivíduos do gênero feminino, idosos, grau de escolaridade no ensino fundamental incompleto, perda auditiva de grau moderado e perda auditiva do tipo neurossensorial bilateral. Quanto ao uso do AASI, a maioria dos sujeitos utilizava aparelho do tipo retroauricular com adaptação binaural. Quanto ao tempo de uso, mais de $70 \%$ utilizava o AASI pela primeira vez, 38\% o fazia por um período de até 12 meses, e mais de $65 \%$ por um período maior que oito horas diárias. As condutas ajuste do AASI e orientação foram mais frequentes para sujeitos com menor tempo de uso do aparelho, enquanto as condutas assistência técnica e reposição, foram mais comuns para os sujeitos com maior tempo de uso. Conclusão: Conhecer características de pacientes usuários de AASI, assim como os motivos do retorno e condutas geradas, contribui para melhor organização e qualidade do serviço prestado.
\end{abstract}

Descritores: Auxiliares de audição; Pessoas com insuficiência auditiva; Continuidade da assistência ao paciente; Necessidades e demandas de serviços de saúde; Socorro de urgência

\section{INTRODUÇÃO}

Segundo estimativa da Organização Mundial da Saúde, em 2005, o número de pessoas com deficiência auditiva incapacitante no mundo era de 278 milhões ${ }^{(1)}$. No Brasil, dois estudos de base populacional abordaram a prevalência desse tipo de deficiência - um deles, desenvolvido em Canoas (RS), indicou $6,8 \%{ }^{(2)}$; o outro, realizado em Monte Negro (RO), apontou 4,8\%(3).

Trabalho desenvolvido no Programa de Estudos Pós-Graduados em Fonoaudiologia, Pontifícia Universidade Católica de São Paulo - PUC-SP - São Paulo (SP), Brasil, com bolsa concedida pela Coordenação de Aperfeiçoamento de Pessoal de Nível Superior - CAPES.

(1) Programa de Estudos Pós-Graduados em Fonoaudiologia, Pontifícia Universidade Católica de São Paulo - PUC-SP - São Paulo (SP), Brasil.

(2) Centro Audição na Criança, Divisão de Educação e Reabilitação dos Distúrbios da Comunicação, Pontifícia Universidade Católica de São Paulo -PUC-SP - São Paulo (SP), Brasil.

(3) Centro Audição na Criança, Divisão de Educação e Reabilitação dos Distúrbios da Comunicação, Pontifícia Universidade Católica de São Paulo - PUC-SP - São Paulo (SP), Brasil.

Endereço para correspondência: Beatriz Cavalcanti de Albuquerque Caiuby Novaes. Centro Audição na Criança - Divisão de Educação e Reabilitação dos Distúrbios da Comunicação - CeAC/DERDIC/PUC-SP. R. Estado de Israel, 860, Vl. Clementino, São Paulo (SP), Brasil, CEP: 04022-040. E-mail: beatriznovaes@pucsp.br

Recebido em: 12/11/2010; Aceito em: 30/4/2011
Em nosso país, o empenho de profissionais da área da saúde, culminou na instituição, pelo Ministério da Saúde (MS), da Política Nacional de Atenção à Saúde Auditiva (PNASA) por meio da Portaria ${ }^{\circ}$ 2073/GM, em 2004, possibilitando, assim, $o$ atendimento integral aos deficientes auditivos ${ }^{(4)}$.

A necessidade de instituição da Política emergiu, principalmente, após constatação, pelo MS, do desequilíbrio entre o número de procedimentos referentes ao diagnóstico e a concessão do aparelho de amplificação sonora individual (AASI), em comparação com a produção ambulatorial dos procedimentos de acompanhamento e reabilitação auditiva ${ }^{(5)}$.

Sendo assim, para que os pacientes do Sistema Único de Saúde (SUS) recebam o AASI por meio de um Serviço de Atenção à Saúde Auditiva (SASA) credenciado, a PNASA propõe uma série de procedimentos a serem seguidos a partir da entrada no serviço; e o acompanhamento é um deles.

O acompanhamento é um procedimento periódico que consiste em avaliações otorrinolaringológicas e audiológicas, com os seguintes objetivos: verificar possíveis alterações nas características audiológicas do paciente, necessidades de ajustes nos moldes auriculares ou nas características eletroacústicas do AASI, e orientar o paciente e seus familiares quanto ao uso desse dispositivo. A periodicidade do acompanhamento depende da faixa etária do indivíduo. 
Após seis anos da implantação da PNASA, realizar o acompanhamento previsto para os usuários de AASI tem sido o grande desafio. A cada ano, conforme mais AASI são concedidos, cresce o número de pacientes que regressam ao serviço, gerando atrasos nos agendamentos de retornos. Além disso, algumas demandas como troca de tubo do molde, confecção de novos moldes, orientação de uso e manuseio e pequenos ajustes no AASI, poderiam ser resolvidas rapidamente em benefício do usuário.

Desta maneira, conhecer as características dos pacientes, os motivos do retorno que geram solicitações de agendamento e fornecer atendimentos imediatos, levaram à organização de uma agenda de Pronto Atendimento (PA), no Serviço de Audiologia Educacional (SAE) da Divisão de Educação e Reabilitação dos Distúrbios da Comunicação (DERDIC).

Vale ressaltar que o PA não elimina a necessidade do acompanhamento, mas permite a pronta resolução, ou encaminhamento para sanar problemas relacionados ao uso do AASI. Evita esperas e muitas vezes é a garantia do uso contínuo do dispositivo de amplificação.

Os objetivos desse estudo foram: estabelecer características demográficas, audiológicas e referentes ao uso de AASI de sujeitos que solicitam retorno a DERDIC; identificar e analisar os motivos do retorno e as condutas geradas e relacionar as condutas de maior ocorrência com as seguintes variáveis: tempo de uso do AASI (meses) e intervalo de tempo entre a data da última avaliação audiológica e a data do PA.

\section{MÉTODOS}

Foi realizado um estudo quantitativo descritivo, de corte transversal, por um período de quatro meses. Todos os participantes assinaram um termo de consentimento livre e esclarecido. Este estudo foi aprovado pelo Comitê de Ética em Pesquisa Pontifícia Universidade Católica de São Paulo (PUC-SP) sob parecer 055/2010.

A pesquisa foi realizada no Serviço de Audiologia Educacional (SAE) da Divisão de Educação e Reabilitação dos Distúrbios da Comunicação (DERDIC), que é a unidade responsável pelos procedimentos: seleção e indicação do AASI, verificação, validação e orientação ao paciente usuário de AASI; terapia de adaptação do dispositivo, acompanhamento e o PA.

Foram incluídos pacientes que: concluíram o diagnóstico audiológico de deficiência auditiva e receberam o AASI na instituição; tinham idade acima de 3 anos; contataram o serviço, por telefone, solicitando retorno e compareceram ao PA na data agendada. A coleta incluiu todos os sujeitos agendados em um período de quatro meses, totalizando 440 pacientes.

As características demográficas e audiológicas dos participantes foram coletadas por meio de pesquisa em prontuários e no sistema de informação da instituição. Os dados referentes ao uso do AASI, motivo do retorno e condutas geradas, foram coletados durante o PA e anotados em ficha específica para tal atendimento.

Os profissionais da recepção do serviço foram orientados quanto aos objetivos do trabalho, pois eram responsáveis pelos agendamentos dos pacientes. Os atendimentos ocorriam de 15 em 15 minutos, em dias e horários específicos e realizados pelo pesquisador e por fonoaudiólogas do serviço.

As informações coletadas na ficha de PA foram registradas em planilha contendo as variáveis incluídas na análise. Os dados foram tratados a partir de estatística descritiva para caracterização dos sujeitos e inferencial para análise de relações. A análise dos resultados orientou-se por três eixos:

- Caracterização demográfica, audiológica e quanto ao uso do AASI (gênero; faixa etária; nível de escolaridade(6); $\operatorname{grau}^{(7)}$ e tipo da perda auditiva; tipo do AASI; tipo de adaptação do AASI; uso ou não do AASI pela primeira vez; tempo de uso do AASI em meses, tempo de uso do AASI em horas diárias e intervalo de tempo entre a data da última avaliação audiológica e a data do PA).

- Análise estatística descritiva dos motivos do retorno, condutas geradas, tempo de uso do AASI (em meses) e intervalo entre as datas da última avaliação audiológica e dia do PA.

- Análise estatística inferencial ${ }^{(8)}$ para associação das condutas geradas com o tempo de uso do AASI (meses) e com o intervalo de tempo decorrido entre a última avaliação audiológica e o dia do PA (meses) por meio do método de Tukey, utilizada para obtenção de informações a partir da análise de medidas simultâneas de muitas variáveis.

\section{RESULTADOS}

Ao todo, 524 sujeitos solicitaram retorno ao serviço, porém, 69 (13\%) faltaram no dia do agendamento e 15 (3\%) foram excluídos; dez por utilizarem AASI concedido por outro SASA e cinco por não possuírem o dispositivo de amplificação. Estes cinco solicitaram nova avaliação audiológica para benefício do passe-livre. Assim, participaram do estudo 440 (84\%) sujeitos.

Do total de sujeitos $(n=440), 258(58,6 \%)$ eram do gênero feminino, $169(38,4 \%)$ tinham idade igual ou superior a 60 anos, 207 (47\%) com perda auditiva de grau moderado na melhor orelha (para o cálculo do grau da perda auditiva na melhor orelha, utilizamos a média de $500 \mathrm{~Hz}, 1 \mathrm{kHz}, 2 \mathrm{kHz}$ e $4 \mathrm{kHz}), 374$ (85\%) com perda auditiva do tipo neurossensorial bilateral e 251 (57\%) com o intervalo de tempo entre a data da última avaliação audiológica e a data do PA (meses) superior a um ano. Em relação ao nível de escolaridade, optamos por considerá-lo apenas para os sujeitos com idade igual ou superior a 15 anos $(n=349)$, pois indivíduos com até 14 anos de idade, encontram-se regularmente em níveis de escolaridade compreendidos até o Ensino Fundamental. Com isso, 141 $(40,4 \%)$ possuíam o ensino fundamental incompleto.

Quanto ao uso do AASI, 388 (88,2\%) utilizavam aparelho do tipo retroauricular, $379(86,1 \%)$ com adaptação binaural, $311(70,7 \%)$ utilizavam seu primeiro aparelho de amplificação, $168(38,1 \%)$ utilizavam o dispositivo por um período de até 12 meses contados a partir da data do recebimento e $288(65,5 \%)$ referiram uso diário do AASI por oito horas ou mais.

Foi criada uma legenda com códigos que representam as categorias de motivo do retorno e condutas geradas para facilitar a leitura ao longo do texto. (Quadro 1).

Da população estudada $(n=440)$, analisamos $386(87,7 \%)$ sujeitos que apresentaram combinação entre motivo do retorno e conduta gerada de maior ocorrência (Tabela 1). 
Quadro 1. Códigos para motivo do retorno e conduta gerada

\begin{tabular}{|l|c|c|}
\hline Código & Motivo do retorno* $^{*}$ & Conduta \\
\hline Ac & Acompanhamento & Acompanhamento \\
Ass & Assistência técnica** & Assistência técnica \\
M & Molde & Molde \\
$\mathrm{Aj}$ & Ajuste no AASI & Ajuste no AASI \\
O & Orientação & Orientação \\
Rep & - & Reposição do AASI \\
Orl & Avaliação & Avaliação \\
& otorrinolaringológica & otorrinolaringológica \\
Laudo & Laudo médico & - \\
Perda & Perda do AASI & - \\
\hline
\end{tabular}

* Muito embora a maioria dos pacientes não utilizasse os termos do quadro acima no momento de expor o motivo do retorno, resolvemos normatizá-los desta forma para melhor compreensão do leitor.

** No caso de motivo do retorno por Assistência técnica, entenda-se AASI danificado.

Para o grupo de 386 sujeitos, apresentamos os valores estatísticos para o tempo de uso do AASI (meses) para cada categoria de conduta gerada no PA. Podemos observar que as condutas de $\mathrm{Aj}$ e $\mathrm{O}$ foram mais ocorrentes em pacientes com tempo de uso do AASI entre 5 e 19 meses, já indivíduos com maior tempo de uso do AASI, variando entre 32 a 53 meses. Esses pacientes retornaram ao serviço por motivo de quebra do aparelho, aumentando o número de encaminhamentos para
Ass e Rep (Tabela 2). As tendências para essa relação também são expressas por meio de box-plot (Figura 1).

Para o mesmo grupo apresentamos os valores estatísticos para o intervalo de tempo entre a data da última avaliação audiológica e a data do PA (meses) para cada categoria de conduta. As condutas de $\mathrm{Aj}$ e $\mathrm{M}$ foram mais ocorrentes em pacientes cujo intervalo de tempo decorrido entre a última avaliação audiológica e a data do PA variou entre sete e 12 meses. Ac, Ass e $\mathrm{M}$ foram condutas mais comuns em pacientes cujo intervalo variou entre 18 e 32 meses (Tabela 3). As tendências para essa relação também são expressas por meio de box-plot (Figura 2).

Comparamos as médias do tempo de uso do AASI e intervalo entre a avaliação audiológica e o PA nas sete condutas geradas, por meio de análise estatística pelo método de Tukey e apresentamos os valores de $\mathrm{p}$ (Tabelas 4 e 5 , respectivamente).

Verificamos a impossibilidade em formarmos grupos disjuntos (que não contenham elementos comuns) de condutas. Por exemplo, observamos que não há diferença entre as médias em Ac+Ass e Ac+M, porém a média em Ac+Ass é diferente da média em Ass, mas não há diferença entre as médias em Ac+M e Ass (Tabela 5).

Os resultados sugerem que as condutas podem, de forma aproximada, ser divididas em dois grupos. O grupo 1, no qual prevalecem as condutas: Ac, Ac+Ass, Ac+M, Ass e M; grupo 2, no qual são notadas as ações: $\mathrm{Aj}$ e $\mathrm{O}$. $\mathrm{O}$ grupo 1 tende a apresentar maiores tempos de uso que o grupo 2 (Tabela 4).

Tabela 1. Distribuição de frequências conjuntas entre motivo do retorno e conduta $(n=386)$

\begin{tabular}{|c|c|c|c|c|c|c|c|c|c|c|c|c|c|c|}
\hline \multirow{2}{*}{ Conduta } & \multicolumn{12}{|c|}{ Motivo } & \multirow{2}{*}{ Total } & \multirow{2}{*}{$\%$} \\
\hline & $\mathrm{Aj}$ & $M$ & Ass & $A c$ & 0 & $A j+M$ & Orl & Laudo & $M+$ Ass & Perda & $A j+A s s$ & $A j+A c$ & & \\
\hline$A c$ & 17 & 8 & 1 & 30 & & 1 & 1 & 8 & & 1 & & & 67 & 17,4 \\
\hline$A c+A s s$ & 11 & 4 & 21 & 10 & & 1 & 2 & 1 & & & 3 & 2 & 55 & 14,2 \\
\hline M & & 47 & & & & 1 & & & & & & & 48 & 12,4 \\
\hline Ass & 10 & & 28 & 3 & & & & & & & 2 & & 43 & 11,1 \\
\hline $\mathrm{Aj}$ & 37 & & & & & 1 & & & & & & 1 & 39 & 10,1 \\
\hline $\mathrm{Ac}+\mathrm{M}$ & & 27 & & 2 & & 1 & & & 1 & & & & 31 & 8,0 \\
\hline $\mathrm{O}$ & 4 & 3 & 2 & 2 & 12 & & & & & 2 & & & 25 & 6,5 \\
\hline $\mathrm{Ac}+\mathrm{O}$ & 5 & & & 2 & 4 & 1 & & & & 2 & & & 14 & 3,6 \\
\hline$A c+$ Ass $+M$ & 3 & 8 & 2 & & & 1 & & & & & & & 14 & 3,6 \\
\hline$A c+A j$ & 8 & & & & & 1 & & & & & & 1 & 10 & 2,6 \\
\hline Rep & & & 6 & & & & & & & 2 & & & 8 & 2,1 \\
\hline Orl & & 1 & & & & & 6 & & & & & & 7 & 1,8 \\
\hline Ass+M & & 1 & & & & 1 & & & 4 & & & & 6 & 1,6 \\
\hline $\mathrm{Ac}+\mathrm{Ass}+\mathrm{O}$ & 1 & & & 2 & & & & & 1 & & & & 4 & 1,0 \\
\hline$M+A j$ & 1 & & & & & 3 & & & & & & & 4 & 1,0 \\
\hline$A c+$ Ass+Rep & 1 & & 1 & & & & & & 1 & & & & 3 & 0,8 \\
\hline$A c+M+A j$ & 1 & & & 1 & & 1 & & & & & & & 3 & 0,8 \\
\hline $\mathrm{Ass}+\mathrm{O}$ & 1 & & & & & 1 & & & & & & & 2 & 0,5 \\
\hline$A s s+A j$ & 2 & & & & & & & & & & 1 & & 3 & 0,8 \\
\hline Total & 102 & 99 & 61 & 52 & 16 & 14 & 9 & 9 & 7 & 7 & 6 & 4 & 386 & 100,0 \\
\hline$\%$ & 26,4 & 25,6 & 15,8 & 13,5 & 4,1 & 3,6 & 2,3 & 2,3 & 1,8 & 1,8 & 1,6 & 1,0 & 100,0 & \\
\hline
\end{tabular}

Legenda: $A c$ = acompanhamento; Ass = assistência técnica; $\mathrm{M}=$ molde; $\mathrm{Aj}$ = ajuste no AASI; O = orientação; Rep = reposição do AASI; Orl = avaliação otorrinolaringológica; Laudo = laudo médico; Perda $=$ perda do AASI 
Tabela 2. Estatística descritiva para o tempo de uso do AASI (meses) em cada categoria de conduta $(n=386)$

\begin{tabular}{|c|c|c|c|c|c|c|}
\hline Conduta & $\mathrm{n}$ & Média & $\mathrm{DP}$ & Mínimo & Mediana & Máximo \\
\hline$A c+$ Ass + Rep & 3 & 53,7 & 23,5 & 30 & 54 & 77 \\
\hline Rep & 8 & 43,9 & 15,2 & 20 & 46,5 & 66 \\
\hline$A c+A s s+M$ & 14 & 37,9 & 19,1 & 11 & 35 & 89 \\
\hline Ass & 43 & 33,4 & 25,5 & 1 & 30 & 88 \\
\hline$A c+A s s$ & 55 & 32,5 & 25,5 & 4 & 27 & 92 \\
\hline Ass $+M$ & 6 & 32,3 & 23,7 & 5 & 27,5 & 60 \\
\hline$A c+O$ & 14 & 30,6 & 24,6 & 4 & 23 & 72 \\
\hline Orl & 7 & 28,6 & 23,7 & 9 & 25 & 77 \\
\hline$A c+M$ & 31 & 27,3 & 17,7 & 2 & 27 & 75 \\
\hline M & 48 & 26,7 & 22,1 & 1 & 20,5 & 89 \\
\hline$A c$ & 67 & 26,5 & 23,1 & 1 & 18 & 91 \\
\hline$A c+A j$ & 10 & 19,0 & 15,8 & 6 & 11,5 & 42 \\
\hline$A c+M+A j$ & 3 & 17,7 & 2,1 & 16 & 17 & 20 \\
\hline$A c+A s s+O$ & 4 & 17,5 & 19,7 & 7 & 8 & 47 \\
\hline$M+A j$ & 4 & 17,3 & 17,2 & 2 & 13,5 & 40 \\
\hline $\mathrm{Aj}$ & 39 & 15,8 & 15,9 & 1 & 9 & 58 \\
\hline $\mathrm{O}$ & 25 & 10,8 & 15,5 & 1 & 5 & 64 \\
\hline$A s s+A j$ & 3 & 10,7 & 10,0 & 3 & 7 & 22 \\
\hline Ass +0 & 2 & 5,0 & 0,0 & 5 & 5 & 5 \\
\hline Total & 386 & 26,7 & 22,6 & 1 & 20 & 92 \\
\hline
\end{tabular}

Legenda: $\mathrm{Ac}=$ acompanhamento; Ass = assistência técnica; Rep = reposição; $\mathrm{M}=$ molde; $\mathrm{O}=$ orientação; Orl = avaliação otorrinolaringológica; $\mathrm{Aj}=$ ajuste no AASI; DP = desvio-padrão

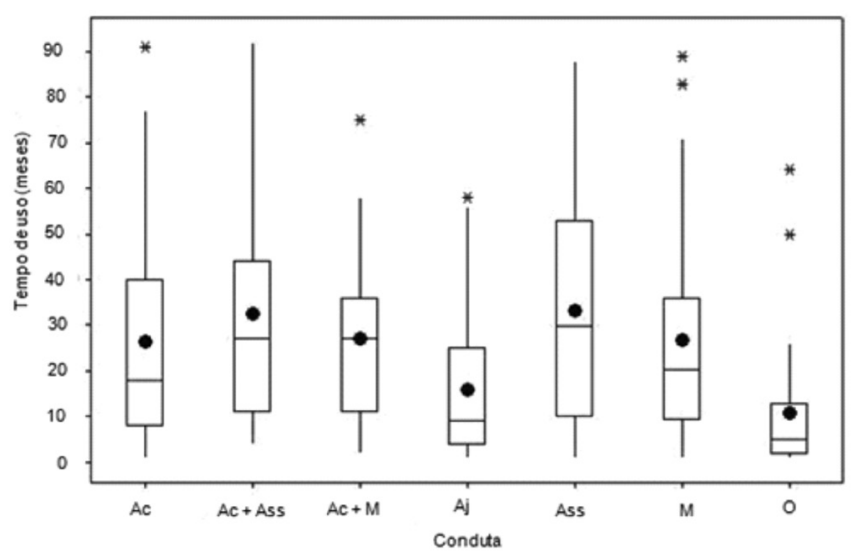

Legenda: $A c=$ acompanhamento; $A s s=$ assistência técnica; $M=$ molde; $A j=$ ajuste no $\mathrm{AASI} ; \mathrm{O}=$ orientação

Figura 1. Box-plots para o tempo de uso do AASI (meses) em cada conduta

Quando considerado o intervalo entre a avaliação audiológica e o pronto atendimento, as condutas podem, de forma aproximada, ser divididas em três grupos, conforme segue: grupo 1 - Ac e Ac+Ass; grupo 2 - Ac+M e Ass; grupo $3-\mathrm{Aj}$, $\mathrm{M}$ e O. As médias do intervalo diminuem do grupo 1 para o grupo 3 (Tabela 5).

Para obter uma regra que permita classificar um indivíduo em um desses grupos, foi aplicada a técnica de análise discriminante. Consideramos um único agrupamento de condutas, de forma a construir uma regra de classificação baseada nas duas variáveis: tempo de uso e intervalo entre a última avaliação audiológica e o PA. O agrupamento considerado ocorreu conforme segue: grupo $1-\mathrm{Ac}$, Ac+Ass, Ac+M e Ass, e grupo $2-\mathrm{Aj}, \mathrm{M}$ e $\mathrm{O}$.

O grupo 1 tende a apresentar maiores tempos de uso e maiores intervalos entre a avaliação audiológica e o PA. A regra de classificação obtida foi: classificar um indivíduo no grupo 1 se: 0,6 x sqrt (intervalo) $+0,2$ x sqrt (tempo) $>3$; caso contrário, classificar o indivíduo no grupo 2, onde: sqrt (intervalo) = raíz quadrada do intervalo entre a avaliação audiológica e o PA e sqrt (tempo) $=$ raíz quadrada do tempo de uso. Esta regra de classificação classificou corretamente $221(71,8 \%)$ indivíduos da amostra $(n=386)$.

\section{DISCUSSÃO}

Embora a faixa etária da população estudada, tenha variado entre 3 e 98 anos (média de 45,9 anos), os indivíduos acima de 60 anos corresponderam a quase $40 \%$ dela. Um estudo com 2.143 indivíduos de mesma faixa etária mostrou que a autopercepção ruim da audição parece aumentar com a idade, e que o impacto negativo causado faz com que o indivíduo e/ ou sua família busquem ajuda ${ }^{(9)}$.

Observa-se também a predominância do gênero feminino, considerado pela literatura como o de maior longevidade ${ }^{(10,11)}$. As mulheres manifestam maior preocupação com a deterioração da audição, assim como com a saúde geral, quando comparadas aos homens ${ }^{(12)}$.

$\mathrm{O}$ ensino fundamental incompleto predominou entre os 
Tabela 3. Estatística descritiva para o intervalo de tempo entre a data da última avaliação audiológica e a data do PA (meses) em cada categoria de conduta $(n=386)$

\begin{tabular}{|c|c|c|c|c|c|c|}
\hline Conduta & $\mathrm{n}$ & Média & $\mathrm{DP}$ & Mínimo & Mediana & Máximo \\
\hline$A c+A s s+R e p$ & 3 & 32,7 & 10,9 & 22,6 & 31,3 & 44,2 \\
\hline$A c+A s s+M$ & 14 & 24,8 & 13,7 & 11,0 & 17,1 & 49,5 \\
\hline$A c+M+A j$ & 3 & 24,2 & 5,4 & 18,0 & 26,4 & 28,2 \\
\hline$A c+$ Ass & 55 & 23,9 & 16,6 & 6,1 & 17,2 & 84,5 \\
\hline$A c$ & 67 & 21,2 & 15,2 & 3,6 & 16,6 & 82,8 \\
\hline Ass $+O$ & 2 & 20,4 & 10,9 & 12,7 & 20,4 & 28,1 \\
\hline$A c+A j$ & 10 & 18,9 & 9,8 & 4,0 & 18,4 & 41,1 \\
\hline$A c+O$ & 14 & 18,8 & 8,4 & 6,3 & 16,9 & 41,3 \\
\hline$A c+M$ & 31 & 18,1 & 10,2 & 5,1 & 15,8 & 47,6 \\
\hline Ass & 43 & 15,9 & 18,1 & 0,1 & 9,3 & 85,8 \\
\hline$A c+A s s+O$ & 4 & 15,2 & 2,7 & 11,5 & 15,8 & 17,7 \\
\hline Rep & 8 & 13,9 & 14,1 & 4,0 & 11,0 & 47,0 \\
\hline$A s s+A j$ & 3 & 12,1 & 4,2 & 9,1 & 10,3 & 16,8 \\
\hline $\mathrm{O}$ & 25 & 11,7 & 10,1 & 1,4 & 9,6 & 57,4 \\
\hline Orl & 7 & 9,9 & 5,2 & 3,7 & 10,0 & 20,1 \\
\hline Ass $+M$ & 6 & 9,6 & 5,2 & 0,1 & 10,4 & 14,4 \\
\hline $\mathrm{Aj}$ & 39 & 9,5 & 6,3 & 0,3 & 8,2 & 33,1 \\
\hline$M$ & 48 & 7,9 & 6,8 & 0,2 & 6,1 & 29,9 \\
\hline$M+A j$ & 4 & 7,8 & 4,1 & 4,2 & 6,6 & 13,8 \\
\hline Total & 386 & 16,6 & 13,8 & 0,1 & 13,0 & 85,8 \\
\hline
\end{tabular}

Legenda: $A c$ = acompanhamento; Ass = assistência técnica; Rep = reposição; $M=$ molde; $\mathrm{O}$ = orientação; Orl =avaliação otorrinolaringológica; $\mathrm{Aj}=\mathrm{ajuste}$ no $\mathrm{AASI}$; $\mathrm{DP}=$ desvio-padrão

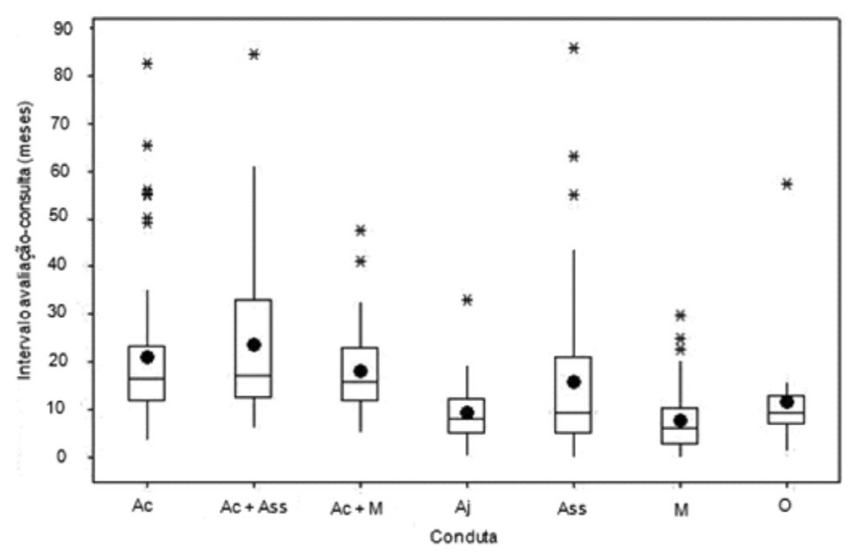

Legenda: $A c=$ acompanhamento; Ass = assistência técnica; $M=$ molde; $A j=$ ajuste no AASI; O = orientação

Figura 2. Box-plots para o intervalo entre a avaliação audiológica e o dia do PA (meses) em cada conduta

sujeitos, fato constatado pela literatura, pois, dentre os indivíduos que procuram os serviços públicos de saúde no país há predominância de baixa escolaridade ${ }^{(13,14)}$.

A ocorrência de perda auditiva do tipo neurossensorial bilateral e de grau moderado, corrobora os achados de outras pesquisas realizadas com usuários de $\mathrm{AASI}^{(15,16)}$, visto que, a indicação de uso do AASI é o meio primário de reabilitação para pacientes com essas características.

Dispositivos retro-auriculares possuem níveis de amplifi-
Tabela 4. Valores de p obtidos na comparação das médias do tempo de uso do AASI (meses) nas condutas, duas a duas

\begin{tabular}{lcccccc}
\hline Conduta & Ac+Ass & Ac+M & $\mathrm{Aj}$ & Ass & $\mathrm{M}$ & $\mathrm{O}$ \\
\hline Ac & 0,695 & 0,998 & 0,092 & 0,733 & 0,999 & $0,003^{*}$ \\
Ac+Ass & & 0,989 & $0,001^{*}$ & 0,999 & 0,885 & $0,001^{*}$ \\
Ac+M & & & 0,082 & 0,988 & 0,999 & $0,003^{*}$ \\
Aj & & & & $0,003^{*}$ & 0,084 & 0,825 \\
Ass & & & & & 0,896 & $0,001^{*}$ \\
M & & & & & & $0,003^{*}$ \\
\hline
\end{tabular}

*Valores significativos $(p<0,05)$ - Teste de Tukey

Legenda: $A c=$ acompanhamento; Ass = assistência técnica; $M=$ molde; $\mathrm{O}=$ orientação; $\mathrm{Aj}$ = ajuste no AASI

Tabela 5. Valores de $p$ obtidos na comparação das médias do intervalo entre a avaliação audiológica e o PA (meses) nas condutas, duas a duas

\begin{tabular}{lcccccc}
\hline Conduta & Ac+Ass & Ac+M & Aj & Ass & $\mathrm{M}$ & $\mathrm{O}$ \\
\hline Ac & 0,939 & 0,963 & $0,001^{*}$ & $0,021^{*}$ & $0,001^{*}$ & $0,009^{*}$ \\
Ac+Ass & & 0,560 & $0,001^{*}$ & $0,001^{*}$ & $0,001^{*}$ & $0,001^{*}$ \\
Ac+M & & & $0,006^{*}$ & 0,545 & $0,001^{*}$ & 0,259 \\
Aj & & & & 0,421 & 0,926 & 0,955 \\
Ass & & & & & $0,021^{*}$ & 0,991 \\
M & & & & & & 0,411 \\
\hline
\end{tabular}

* Valores significativos $(p<0,05)$ - Teste de Tukey

Legenda: $\mathrm{Ac}=$ acompanhamento; Ass = assistência técnica; $\mathrm{M}=$ molde; $\mathrm{O}=$ orientação; $A j$ = ajuste no AASI 
cação importantes, controles maiores e mais visíveis, muitas possibilidades de ajustes e estética aceitável ${ }^{(17,18)}$, sendo os mais indicados quando comparados com os demais tipos. Mais de $85 \%$ dos pacientes faziam uso de adaptação binaural, que proporciona melhor localização sonora e reconhecimento de fala na presença do ruído quando comparada à monoaural ${ }^{(19)}$, possibilitando assim, melhor benefício ao paciente.

Mais de $70 \%$ dos indivíduos que solicitaram retorno ao serviço faziam uso de seu primeiro AASI. Esse tipo de usuário necessita de maior atenção por parte dos fonoaudiólogos, principalmente, no que diz respeito à orientação ${ }^{(20)}$. A produção de material didático em Digital Video Disc (DVD) ${ }^{(21)}$ e a confecção de um guia de orientação ${ }^{(22)}$ foram estratégias criadas por alguns pesquisadores de modo a proporcionar maior esclarecimento aos pacientes.

O presente estudo concluiu que quase $40 \%$ dos indivíduos que solicitaram retorno, eram usuários de AASI por um período de até 12 meses, isso mostra que o primeiro ano após a aquisição do AASI é considerado o período crítico de adaptação ${ }^{(23)}$, no qual há risco de desistência; logo, a identificação de dificuldades e a orientação oportuna a cada caso, diminuem o índice de abandono do uso do AASI $^{(24)}$.

Mais de 65\% dos sujeitos referiu uso diário do AASI por mais de oito horas. Conforme estudo em que utilizou o Practical Hearing Aid Skills Test (PHAST) ${ }^{(25)}$, a quantidade de horas diárias de uso do dispositivo por parte dos pacientes é diretamente proporcional ao resultado no teste no que diz respeito ao domínio do uso de seus AASI, ou seja, quanto mais se utiliza o AASI, maior habilidade quando ao uso do mesmo.

Um total de 57\% dos sujeitos estava com a data da avaliação audiológica vencida, segundo o período preconizado para sua faixa etária conforme a PNASA; isto é, essa avaliação havia sido realizada há mais de seis meses, em crianças de 3 a 14 anos e 11 meses, ou há mais de um ano, em sujeitos com idade igual ou superior a 15 anos. Embora o retorno do paciente muitas vezes demande outro tipo de conduta, como ajuste nos moldes auriculares e orientação ${ }^{(26)}$, a avaliação audiológica realizada no período determinado possibilita, entre outros benefícios, o ajuste, com segurança, dos parâmetros eletroacústicos do AASI.

As maiores ocorrências dentre as combinações de motivo do retorno e conduta, foram aquelas em que motivo e conduta foram iguais, como $\mathrm{Aj}-\mathrm{Aj}(36,3 \%), \mathrm{M}-\mathrm{M}$ (47,5\%), Ass-Ass $(46 \%)$, Ac-Ac (58\%) e O-O (80\%), demonstrando que, muitas vezes, o sujeito que procura o serviço tem consciência do que realmente está precisando naquele momento, e cabe ao serviço organizar-se para receber essa demanda. A demanda diz respeito à atitude do indivíduo de procurar serviços de saúde, e se beneficiar com o atendimento recebido, e a oferta de serviços de saúde está relacionada à disponibilidade, ao tipo e à quantidade de serviços e de recursos destinados a atenção à saúde ${ }^{(27)}$, sendo assim, a relação entre oferta e demanda deve ser sempre buscada.

Quanto às condutas relacionadas ao tempo de uso do AASI, $\mathrm{Aj}$ e $\mathrm{O}$ foram as mais ocorrentes para sujeitos com menor tempo de uso do AASI. Observamos em estudo realizado com indivíduos que faziam uso do AASI há pouco tempo (mínimo de três meses), que as principais queixas eram relacionadas às características de amplificação (intensidade, desconforto etc.) e dificuldade em manusear e limpar os moldes ${ }^{(28)}$. Por outro lado, o fato de haverem indivíduos que retornaram ao serviço e foram encaminhados para Ass e Rep, demonstra que, decorridos seis anos da instituição da PNASA, este fato se torna comum, o que indica, a necessidade de estudos exploratórios sobre a manutenção dos AASI, as falhas técnicas mais frequentes e a razão de suas ocorrências ${ }^{(3)}$.

Quanto às condutas relacionadas ao intervalo de tempo decorrido entre a última avaliação audiológica e a data do PA, observamos que retornos por $\mathrm{Aj}$ e $\mathrm{M}$ foram mais frequentes em pacientes cujo intervalo de tempo variou de 7 a 12 meses. Isso pode ter acontecido durante o período de acompanhamento, ou seja, os pacientes que retornaram para realizá-lo solicitaram retorno para ajuste no AASI e confecção de novos moldes auriculares. Ac, Ass e M foram as condutas mais comuns em pacientes cujo intervalo foi maior, variando de 18 a 32 meses, ou seja, depois de um certo período, podemos perceber que problemas decorrentes do uso contínuo do dispositivo e do molde acarretam retornos para assistência técnica e reposição.

As divisões de grupos de condutas por variáveis analisadas - tempo de uso do AASI e intervalo de tempo entre a última avaliação audiológica e o PA - permite antecipar o atendimento no serviço, por meio das características do motivo do retorno dos pacientes, já que obtivemos a informação sobre aqueles que mais retornam e seus principais motivos. As probabilidades sugerem que quanto maior o tempo de uso, maior a probabilidade da realização de condutas do grupo 1: $\mathrm{Ac}, \mathrm{Ac}+\mathrm{Ass}, \mathrm{Ac}+\mathrm{M}$ e Ass, que incluem aquelas relacionados ao tempo de uso, como necessidade de acompanhamento e assistência técnica. A probabilidade de o usuário gerar condutas do grupo 2: $\mathrm{Aj}, \mathrm{M} \mathrm{e} \mathrm{O}$ foi maior quando o tempo de uso era menor, nos novos usuários, caracterizando o período de adaptação que inclui ajustes no AASI, no molde auricular, ou orientações quanto a manuseio.

O PA demonstrou ser uma estratégia que abrevia a espera da consulta e permite a identificação e atendimento preliminar de demandas dos pacientes. Além disso, o tempo de uso do AASI e o intervalo desde a realização da última avaliação audiológica foram identificados como bons preditores das condutas realizadas e podem orientar o agendamento de consultas, com previsão de tempo de duração e equipamento predeterminados, gerando maior resolução dos problemas. Estes achados reforçam a necessidade de orientação nas etapas iniciais de adaptação ao AASI, garantindo o uso sistemático da amplificação por meio de acompanhamento e reavaliações periódicas ${ }^{(29,30)}$.

\section{CONCLUSÃO}

A obtenção das características (demográficas, audiológicas e relacionadas ao uso do AASI) da população que procurou o serviço permitiu avaliação preliminar do perfil desta população, desde a implementação da PNASA e suas principais demandas. Também foi possível, a partir dos achados, a identificação de possíveis adaptações na organização de processos envolvidos no acompanhamento de usuários de AASI, a saber, organização de agendas diferenciadas na dependência do 
tempo de uso do AASI e do intervalo desde a última avaliação audiológica. No primeiro caso, novos usuários necessitaram, em geral, de orientação, ajustes nos moldes ou nova regulagem. Ao contrário, aqueles que não retornavam há mais de um ano já podem ter sua consulta de acompanhamento agendada antes daquela que avaliará as queixas do usuário com relação ao AASI. O planejamento prévio de demandas específicas do usuário contribui para melhor organização e qualidade do serviço prestado e, uma vez que o crescente número de usuários de AASI acompanhados demanda a criação de novas estratégias de atendimento ao usuário, garante agilidade e consequente satisfação do usuário com o serviço. O presente estudo apresentou limitações na análise de condutas em retornos subsequentes - quando e como os problemas dos pacientes foram resolvidos após a consulta no $\mathrm{PA}$ - pois não compuseram esta etapa da pesquisa. Ficou clara a necessidade do estabelecimento da capacidade do serviço para atender à demanda de acompanhamento e consequente atribuição de horários para este fim. Outros estudos são necessários no âmbito do acompanhamento visto que este tem sido um ponto nevrálgico nas discussões de políticas públicas na saúde auditiva.

\begin{abstract}
Purpose: To establish the demographic and audiological characteristics of subjects users of hearing aids (HA) that requested a return at a Hearing Health Service, and to identify and analyze the reasons for the return and conducts generated during the Emergency Care (EC). Methods: Participants were 440 subjects older than three years, with diagnosis and provision of hearing aids at the service where the research was conducted. Demographic and audiological characteristics and the reason for the use of hearing aids were collected from medical records and specific form used at the EC. Data were analyzed using descriptive and inferential statistics. Results: The studied group was characterized mostly by female individuals, elderly, with incomplete elementary school education, moderate hearing loss, and bilateral sensorineural hearing loss. Most subjects used hearing aids with binaural BTE. As for the time of use, more than $70 \%$ used the hearing aid for the first time, $38 \%$ did so for a period inferior or equal to 12 months, and more than $65 \%$ for more than eight hours a day. The conducts hearing aid adjustment and orientation were more frequent for subjects with shorter use of the device, while the conducts technical assistance and replacement were more common for subjects with longer use. Conclusion: To identify the characteristics of patients users of HA, as well as the reasons of the return and conducts generated by the demand, contributes to better organization and quality of the service provided.
\end{abstract}

Keywords: Hearing aids; Hearing impaired persons; Continuity of patient care; Health services needs and demand; Emergency relief

\title{
REFERÊNCIAS
}

1. World Health Organization. Deafness and hearing impairment [Internet]. 2010 [citado 2010 Maio 23]. Available from: <http://www.who.int/ mediacentre/factsheets/fs300/en/index.html.

2. Béria JU, Raymann BC, Gigante LP, Figueiredo AC, Jotz G, Roithman $\mathrm{R}$, et al. Hearing impairment and socioeconomic factors: a populationbased survey of an urban locality in southern Brazil. Rev Panam Salud Publica. 2007;21(6):381-7.

3. Bevilacqua MC, Melo TM, Morettin M, Lopes AC. A avaliação de serviços em Audiologia: concepções e perspectivas. Rev Soc Bras Fonoaudiol. 2009;14(3):421-6.

4. Brasil. Ministério da Saúde. Política Nacional de Atenção à Saúde Auditiva Portaria $n^{\circ}$ 2073/GM, de 28 de setembro de 2004 [Internet]. 2004 [citado 2010 Mai 23]. Disponível em <http://dtr2001.saude.gov. br/sas/PORTARIAS/Port2004/GM/GM-2073.htm>.

5. Daher CV, Pisaneschi E. A Política Nacional de Atenção à Saúde Auditiva: à atenção especializada às pessoas com deficiência auditiva no SUS. In: Bevilacqua MC, Martinez MA, Balen AS, Pupo AC, Reis AC, Frota S, organizadores. Saúde auditiva no Brasil: políticas, serviços e sistemas. São José dos Campos: Pulso Editorial; 2010. p.15-30.

6. Bertagna RH, Camargo RG. Ensino fundamental de nove anos: análise a partir da experiência de uma escola municipal de Campinas. Cad Pedag. 2009;1(5):242-54.

7. World Health Organization. Prevention of blindness and deafness. Grades of hearing impairment [Internet]. 2011 [citado 2010 Maio 23]. Available from: <http://www.who.int/pbd/deafness/hearing impairment_grades/en/index.html>.

8. Johnson RA, Wichern DW. Applied multivariate statistical analysis. 3rd ed. New Jersey: Prentice-Hall; 1992.
9. Lebrão ML, Cardoso MR; Morettin M. Fatores relacionados à autopercepção da audição entre idosos do município de São Paulo Projeto SABE. Saúde Coletiva. 2008;5(24):168-72.

10. Camarano A. Mulher idosa: suporte familiar ou agente de mudança?. Estud Av. 2003;17(49):35-63.

11. Campos NO, Rodrigues RN. Ritmo de declínio nas taxas de mortalidade dos idosos nos estados do Sudeste, 1980-2000. Rev Bras Estud Populacionais. 2004;21(2):323-42.

12. Espmark AK, Rosenhall U, Erlandsson S, Steen B. The two faces of presbyacusis: hearing impairment and psychosocial consequences. Int J Audiol. 2002;41(2):125-35.

13. Gouveia GC, Souza WV, Luna CF, Souza-Júnior PR, Szwarcwald CL. Health care users' satisfaction in Brazil, 2003. Cad Saúde Pública. 2005;21(Supl 1):S109-18.

14. Ribeiro MC, Barata RB, Almeida MF, Silva ZP. Perfil sociodemográfico e padrão de utilização de serviços de saúde para usuários e não-usuários do SUS - PNAD 2003. Cienc Saúde Coletiva. 2006;11(4):1011-22.

15. Teixeira CF, Augusto LG, Caldas Neto SS. Prótese auditiva: satisfação do usuário com sua prótese e com seu meio ambiente. Rev CEFAC. 2008;10(2):245-53.

16. Silva BS, Sousa GB, Russo IC, Silva JA. Caracterização das queixas, tipo de perda auditiva e tratamento de Indivíduos idosos atendidos em uma clínica particular de Belém-PA. Arq Int Otorrinolaringol. 2007;11(4):387-95.

17. Menegotto IH, Iório MC, Borges AC. Próteses auditivas: relação entre controle de volume e ganho. Pró-Fono. 1993;5(2):8-13.

18. Zandavalli MB, Christmann LS, Garcez VR. Rotina de procedimentos utilizados na seleção e adaptação de aparelhos de amplificação sonora 
individual em centros auditivos na cidade de Porto Alegre, Brasil - RS. Rev CEFAC. 2009;11(Supl 1):106-15.

19. Caetano MH, Marone SA, Ruggieri M. Aparelho de amplificação sonora digital - características e vantagens. Arq Int Otorrinolaringol. 1999;3(3):120-5.

20. Almeida MR, Guarinello AC. Reabilitação audiológica em pacientes idosos. Rev Soc Bras Fonoaudiol. 2009;14(2):247-55.

21. Campos K, Oliveira JR, Blasca WQ. Processo de adaptação de aparelho de amplificação sonora individual: elaboração de um DVD para auxiliar a orientação a indivíduos idosos. Rev Soc Bras Fonoaudiol. 2010;15(1):19-25.

22. Dantas CM. Atendimento domiciliar ao indivíduo idoso novo usuário de aparelhos de amplificação sonora [dissertação]. São Paulo: Pontifícia Universidade Católica de São Paulo; 2009.

23. Tschiedel RS. Programa de reabilitação audiológica para idosos usuários de aparelhos de amplificação sonora individual e seus interlocutores mais frequentes [tese]. Brasília: Universidade de Brasília; 2003.

24. Costa MH, Sampaio AL, Oliveira CA. Avaliação do benefício da prótese auditiva digital e da percepção da desvantagem auditiva. Arq Int Otorrinolaringol. 2007;11(2):159-68.
25. Desjardins JL, Doherty KA. Do experienced hearing aid users know how to use their hearing AIDS correctly? Am J Audiol. 2009;8(1):9-76.

26. Scholze AS, Pagnossim DF, Fialho IM. Acolhimento em audiologia. In: Balen AS, Pagnossim DF, Fialho IM, Zimmermann KJ, Roggia SM, organizadores. Saúde auditiva da teoria à prática. São Paulo: Santos; 2010. p.123-33.

27. Ralo EJ. Qualidade em serviço na saúde auditiva infantil: agendamento, espera e permanência [dissertação]. São Paulo: Pontifícia Universidade Católica de São Paulo; 2010.

28. Freitas CD, Costa MJ. Processo de adaptação de próteses auditivas em usuários atendidos em uma instituição pública federal - parte I: resultados e implicações com o uso da amplificação. Rev Bras Otorrinolaringol. 2007;3(6):744-51.

29. Morettin M. Avaliação dos benefícios e satisfação dos usuários de aparelho de amplificação sonora individual nos serviços de audiologia do SUS. [dissertação]. São Paulo: Universidade de São Paulo; 2008.

30. Carvalho JS. Satisfação de idosos com aparelhos auditivos concedidos no estado de Tocantins. Arq Int Otorrinolaringol. 2007;11(4):416-26. 